\title{
Older people perceived health as going and doing something meaningful
}

\author{
Bryant LL, Corbett KK, Kutner JS. In their own words: a model of healthy aging. Soc Sci Med 2001 Oct;53:927-41
}

\section{QUESTION: What constitutes and contributes to the health of older people?}

Design
Grounded theory type analysis.

Setting

Healthcare facilities of a group practice health maintenance organisation in the US.

\section{Participants}

22 older individuals who were randomly selected from those whose reported perceived health differed from that predicted by a regression model constructed from data from a randomised trial of a primary care intervention.

\section{Methods}

Semistructured interviews lasted approximately 45 minutes and took place in participants' homes or in healthcare facilities. Questions targeted perceptions of

For correspondence:

Dr L Bryant, University

of Colorado Health

Sciences Center, Denver

CO, USA.

lucinda.bryant@uchsc.edu

\section{COMMENTARY}

A major difficulty in studying healthy aging is the definition of health itself. Previous studies that defined health as the absence of chronic disease or physical or cognitive impairment ${ }^{12}$ have failed to identify factors to promote healthy aging that older adults think are important. This qualitative study by Bryant $e t$ al provides important new insight into how older adults define health and what they believe contributes to it.

One strength of this study was the use of an innovative sampling plan. Participants, whose self reported ratings of their perceived health did not match that predicted by their chronic conditions, functional status, and socioeconomic resources, were identified from a clinical trial. Qualitative interviews were used to identify, from the participants' perspectives, views on health and the factors that contribute to it. The findings include factors that contribute to the desired outcome of healthy aging that have not been identified in previous quantitative research. Health was perceived as the ability to go and engage in meaningful activity. Furthermore, people who reported better health than their predicted rating tended to take a more global view of health compared with those who reported worse health than predicted, who tended to focus on physical aspects.

One limitation in the interpretation of these findings is the limited description of the characteristics of the 22 interview participants. Bryant $e t$ al acknowledge that potential participants who refused to be interviewed were older, had more chronic conditions, had lower mobility and physical ability, were more depressed, and had poorer health. More complete reporting of the demographic characteristics and scores on predictor measures would make it possible to judge the transferability of the findings to other older adults.

The study findings offer nurses directions for reframing how they think about healthy aging. Healthy aging includes the whole person, not just the physical body. Older adults who do something meaningful feel healthier than those who do not. Hence, a key question to ask our patients is: "What gives your life meaning and purpose?"

Deborah C Messecar, RN, CS, MPH, PhD Assistant Professor, School of Nursing Oregon Health and Science University Portland, Oregon, USA

1 Burke GL, Arnold AM, Bild DE, et al. Factors associated with healthy aging: the Cardiovascular Health Study. J Am Geriatr Soc 2001;49:254-62.

2 Reed DM, Foley DJ, White LR, et al. Predictors of healthy aging in men with high life expectancies. Am J Public Health 1998;88:1463-8.

health; wellbeing; valued abilities, activities, and relationships; social support; control; sense of coherence; and personal outlook. Analysis of the interviews employed grounded theory type immersion into the material. This process led to the identification of a central phenomenon and the relation of other factors with it.

\section{Main findings}

For these older individuals, health meant going and doing something meaningful. Going meant more than mobility and doing meant more than physical function. Going and doing did not have to involve physical activity, but required 4 components: something worthwhile and desirable to do, balance between abilities and challenges, appropriate external resources, and personal attitudinal characteristics.

Something worthwhile and desirable to do involved activities that were valued such as social activities, travel, reading, housework, fishing, swimming, and creative activities. The number of valued activities identified by an individual did not necessarily relate to a more positive assessment of health. Pain, disability, and depression affected individuals' abilities to identify or engage in meaningful activities.

Balance between abilities and challenges affected how satisfactorily these older individuals could accomplish valued activities. They spoke of mobility, vision, and mental function as particularly important. Remaining independent emerged as an important contributor to better perceived health. Perspectives ranged from being resigned to limitations attributed to aging to a determination to continue to do as much as possible.

Appropriate external resources were necessary to support going and doing. Almost all participants spoke of friends and family when asked about valued relationships. Having a spouse did not guarantee a positive perception of health, especially if the spouse had poor health and required caretaking. Cooperative marriages appeared to support positive perceptions. Social support was only part of what people needed. Participants also spoke of health care as a resource.

Personal attitudinal characteristics emerged as the most important contributor to positive perceived health. Factors thought to contribute to a positive attitude included upbringing, religious faith, and control or willpower. Individuals who expressed positive attitudes, assertiveness, determination to continue to be active, and a desire to take charge perceived their health status more favourably than those who did not.

\section{Conclusion}

Older people perceived healthy aging in terms of going and doing something meaningful, which comprised 4 components. 